\title{
Review
}

Ophthalmic

Research

Ophthalmic Res 2016;55:26-36

DOI: $10.1159 / 000441033$
Received: September 7, 2015

Accepted: September 10, 2015

Published online: November 11, 2015

\section{Metamorphopsia: An Overlooked Visual Symptom}

\author{
Edoardo Midena ${ }^{\mathrm{a}} \mathrm{b}$ Stela Vujosevic ${ }^{\mathrm{a}}$ \\ ${ }^{a}$ Department of Ophthalmology, University of Padova, Padova, and ${ }^{b} \mathrm{G} . B$. Bietti Eye Foundation, Rome, Italy
}

\section{Key Words}

Metamorphopsia - Visual function - Macular disease .

Age-related macular degeneration - Vitreoretinal

interface disorder - Diabetic retinopathy - Central serous

chorioretinopathy

\begin{abstract}
Metamorphopsia is a common symptom in different macular disorders. Micropsia and macropsia are special types of metamorphopsia. Recent theories suggest that both retinal and cortical mechanisms are involved in the development and changes of metamorphopsia. Different functional tests have been proposed for the evaluation of metamorphopsia: from the Amsler grid to the hand-held mobile devices for home monitoring. This review addresses some new insights into the pathophysiology of metamorphopsia and different available tests for the evaluation of this symptom in most common macular disorders. The importance of quantification of metamorphopsia in macular diseases is confirmed by the most recent therapeutic approaches.
\end{abstract}

(c) 2015 S. Karger AG, Basel

\section{Introduction}

Metamorphopsia is a symptom of visual function disturbance in different macular disorders. Some of the most common macular diseases characterized functionally by metamorphopsia include but are not limited to: age-related macular degeneration (AMD), diabetic and nondiabetic macular edema, vitreoretinal interface disorders, retinal detachment, central serous chorioretinopathy (CSC), and any macular serous elevation. Even though it was thought that metamorphopsia is a pure consequence of structural changes in the retina, resulting for the displacement of more external retinal layers with improper light signal transduction, some recent theories have emphasized the importance of the combination of more internal retinal and sometimes cortical processing [1]. The Amsler grid was the first functional test proposed to evaluate metamorphopsia [2]. The Amsler grid allows for simple and rapid qualitative evaluation of alterations of visual function in the central $10^{\circ}$, which may precede or accompany the beginning and evolution of some macular disorders. Because the standard Amsler grid is a supra-

\section{KARGER}

E-Mail karger@karger.com www.karger.com/ore (c) 2015 S. Karger AG, Base

0030-3747/15/0551-0026\$39.50/0
Prof. Edoardo Midena, MD, PhD

Department of Ophthalmology University of Padova

Via Giustiniani 2, IT-35128 Padova (Italy)

E-Mail edoardo.midena @ unipd.itt 
threshold test stimulus and is not sensitive enough in early detection of metamorphopsia, many more different sensitive tests have been proposed for early detection and quantification of metamorphopsia, but most of them not fully clinically validated. Differential perimetry, MCHARTS, preferential hyperacuity, and shape discrimination hyperacuity tests have been used for the evaluation of metamorphopsia. Moreover, in order to facilitate home monitoring of the disease, and thus allow for earlier diagnosis and treatment, with possible better functional outcome, several home-monitoring devices based on preferential hyperacuity testing have been proposed. The aim of this review is to analyze the different tests used to detect and follow metamorphopsia, and evaluate the use of these tests in different macular disorders for quantification of metamorphopsia and follow-up after therapeutic intervention.

\section{Definition and Classification of Metamorphopsia}

Metamorphopsia was first described by Forster [3], then by Knapp [4] and Wundt [5], in the 19th century as one of the earliest and most characteristic signs of myopic and senile maculopathies. Subsequently, Amsler [6,7] described and defined in detail (1949 and 1953) this visual symptom. Metamorphopsia was defined as the deviation of either vertical or horizontal lines, reported by the patient, and consisted of the first visual disturbance sometimes preceding clinical appearance of maculopathy $[6$, 7]. Sometimes, metamorphopsia is perceived by the patient as an 'instability' of vision, instead of precise deformation of objects, and is brought about by slight unconscious and involuntary movements of the eye in an effort to better focalize the object [7]. Special types of metamorphopsia include micropsia and macropsia. Micropsia, which is more common, is a perception of objects smaller than they actually are, whereas the rarer macropsia is the perception of objects larger than they actually are.

Amsler [7] emphasized the importance of early detection of the cause of disturbances of vision, which may not be visible on ophthalmoscopic examination of the fundus and cannot be detected by standard quantitative examination of visual function, such as a visual acuity test. Normal visual acuity and normal fundus appearance may be associated with functional disturbances, as reported by the patient, and a maculopathy may be present [7]. Thus, the importance of the patients' visual function symptoms, which are often underestimated in clinical practice, is relevant in the diagnosis of macular disorders. Metamor- phopsia also dramatically deteriorates reading abilities and face perception, significantly contributing to the deterioration of the quality of life of affected patients. Moreover, metamorphopsia, which is extremely disabling to the patient, may represent the best way to monitor the functional results of any treatment of macular disorders, especially in the era of intravitreal therapies and macular microsurgery.

\section{Pathophysiology of Metamorphopsia}

Since the 19th century, the origin of metamorphopsia was considered to purely depend on displacement of the perceptual elements (cones) of the retina and a false localization of the image seen by these displaced elements $[3,8]$. In fact, Forster [3] reported that macropsia is the outcome of retinal contraction, as parallel lines are separated from each other by an outward curve (meaning that retinal elements are approximated to each other). The opposite is found in micropsia [9-12]. Fluid raises and distends the basal attachment of the cones and separates them so that fewer perceptive elements are stimulated, and therefore objects are seen smaller than they are [912]. Amsler [7] suggested that changes in the outer retina and the choroid are the pathophysiologic drivers of both metamorphopsia and scotoma. Moreover, the same author pointed out that the morphologic basis of metamorphopsia cannot be adequately detected by histopathology, as death, chemical fixation of the eye, and the manipulation in the laboratory destroys or significantly modifies the structure of the sensory elements of the retina in the macular region [7]. With the advent of spectral domain optical coherence tomography (OCT), a detailed, layerby-layer, in vivo evaluation of the retina was obtained. Thus, a detailed correlation between metamorphopsia and OCT findings has been reported, partly confirming a previous hypothesis that changes at the photoreceptor level are the main cause of metamorphopsia. However, Watanabe et al. [13] reported that the inner nuclear layer (INL) was thicker in patients with epiretinal membranes (ERM) who reported broad metamorphopsia than in patients with ERM without metamorphopsia. Okamoto et al. [14] reported the relationship between metamorphopsia and foveal microstructure in patients with ERM. The severity of metamorphopsia was mainly influenced by the thickening of INL and ganglion cell layer, but not by the changes of the outer retina [14]. Although the exact mechanisms by which the thickening of the inner retina leads to metamorphopsia are not completely clear, these 
authors suggested that this phenomenon might be due to changes in horizontal, bipolar, amacrine, and especially Müller cell bodies, all located in the INL. Thus, the normal function of synaptic junctions is inhibited and photoreceptor (PR) sensitivity is decreased, causing metamorphopsia. In addition, the thickening of the ganglion cell layer and INL itself may induce a decrease in the quality of vision [14]. Activated and hypertrophic Müller cells (e.g. after partial detachment of the vitreous from the retina) have a major role in epiretinal membrane formation together with vitreous fibers adhering to Müller cells at sites of vitreoretinal attachment [15]. In this way, Müller cells contribute to the formation of gliotic scars and also to increased vascular leakage by releasing growth factors. Agte et al. [16] also reported on the influence of Müller cell shape and direction concerning the quality of light transduction, indirectly underlining the importance of Müller cells in the pathophysiology of metamorphopsia.

Wiecek et al. [1] suggested that metamorphopsia is not only caused by displacement of retinal layers resulting in mislocation of light on the retina, but also by the combination of retinal changes with cortical processing, mainly after long-standing maculopathy or after treatment of macular disorders (as neovascular AMD). As a consequence, metamorphopsia may also be influenced by information about the scene/stimulus, top-down information, and cortical reorganization.

\section{The Detection of Metamorphopsia}

Amsler recognized the inadequacy of standard perimetry in detecting metamorphopsia or central relative scotomas, and therefore the necessity of a new functional test in order to detect it. In 1947, he proposed his test to record metamorphopsia and relative scotoma, known as the Amsler grid [2].

\section{Amsler Grid}

The Amsler grid consists of a black card or school-slate on which white vertical and horizontal parallel lines are drawn, subdivided every $5 \mathrm{~mm}$, and forming a perfectly regular square of $10 \mathrm{~cm}$. When the grid is presented at $28-30 \mathrm{~cm}$, each small $5-\mathrm{mm}$ square subtends an angle of $1^{\circ}$. The entire grid is thus $20^{\circ}$ high and $20^{\circ}$ wide, $10^{\circ}$ on each side of the center. Therefore, the grid explores the central $10^{\circ}$ of the retina, around the fixation point. The blind spot lies outside the grid approximately $5^{\circ}$ outside the nasal limit. The grid is presented under illumination and after careful refractive correction of the eye. The patient must fix the central point of the grid and not let his eye wander. The patient should be encouraged to do so during the whole examination and should be helped in this by repeated comparison with the other eye. Thereafter, the following questions to the patient are fundamental in order to bring out the perception of metamorphopsia: 'Are the lines which cross in the grid straight and absolutely parallel from beginning to end, especially near the center?', and 'Are all the small squares regular and perfectly equal?'. The patient should localize and describe the sites of metamorphopsia. It is easier for the patient to describe its characteristics if they are localized near the fixation point. There can be various forms of metamorphopsia as detected by the Amsler grid: lines can be broken into small angular irregularities, or curved into smaller or greater ondulations. Forster [3] and Mackay [8] were the first to describe the so-called 'oriented metamorphopsia', which consists of central or paracentral ondulations of only vertical or horizontal lines [3, 8]. Micropsia consists of vertical and horizontal lines curved inwards toward the central point, whereas in macropsia the lines curve away from the center. Diffuse metamorphopsia is defined as a generalized deformation of the entire grid to such an extent that its margins are no longer parallel and the internal lines bend into large waves [7].

Moreover, a correlation between different types of metamorphopsia and different maculopathies has been reported: small angular line defects with myopic maculopathy, ondulations with AMD, diffuse metamorphopsia with retinal detachment, and diffuse irregularity of the grid (as if was drawn by an unsteady hand) even after postsurgical retinal reattachment [7].

Besides metamorphopsia, Amsler [7] proposed his grid for the evaluation of relative scotoma. He described relative (called translucid) scotoma as a translucid spot which does not completely hide the crossing lines of the grid, but covers them with a veil more or less homogeneously. It is usually central and circular, but can also be eccentric. The more distant these spots are from the center of the grid, the more difficulty the patient has in determining their shape. Metamorphopsia and relative scotomas can coexist. Amsler grid testing is used in clinical practice for detecting and monitoring functional impairment due to numerous chorioretinal diseases, such as inherited, vascular and degenerative maculopathies; toxic maculopathies; retinal detachment and macular holes, and optic neuropathies [17-32]. The Amsler grid is also provided to patients for home monitoring [33-40]. How-
28

Ophthalmic Res 2016;55:26-36

DOI: $10.1159 / 000441033$
Midena/Vujosevic 
ever, home monitoring with the Amsler grid has several limitations such as the need for reasonable reading vision to discern the lines, no fixation control during the test, the noninteractive nature and impossibility to quantify metamorphopsia (difficulty in the monitoring at home during the follow-up), low sensitivity and poor performance when evaluating multiple lines that are peripherally presented (due to 'crowding effect'), and limited awareness of visual field defects until the scotoma size is large and involves the fovea (partially due to cortical completion 'filling-in phenomenon') [41, 42].

A possible explanation for the Amsler grid's low sensitivity in detecting scotomas compared to scanning laser ophthalmoscopy perimetry (early microperimetry) may be due (besides the suprathreshold strategy and fixation instability) to a perceptual completion phenomenon which consists of a filling-in of the missing information when part of an image falls on a blind area of visual field $[41,43]$. The mechanisms involved in the completion phenomenon have been studied in animals with electrophysiologic experiments which evaluated correspondence between the receptive fields in the cortical areas and retinal lesions $[44,45]$. The size of the retinal lesion seems crucial for the extent of the cortical completion. In fact, scotomas that are equal to or less than $6^{\circ}$ are poorly detected by the Amsler grid [17]. Moreover, Achard et al. [41] suggested an important role of perceptual completion in rapid change of visual search strategies in patients with central retinal lesions due to the constant change of size and location of scotoma. When the scotoma is located between the fixation point and the target, saccades might be inaccurate because of an altered perception of distances [41]. Therefore, Achard et al. [41] concluded that in AMD, the Amsler grid is useful for detection of metamorphopsia, but is less efficient for determination of the extent of scotoma.

\section{Threshold Amsler Grid Testing}

The Amsler grid is a suprathreshold stimulus and therefore it may not be sensitive enough in early detection of metamorphopsia, as it is analogous to mapping the visual island with a stimulus of high luminance [18, 32]. Therefore, some modifications of the standard Amsler grid have been proposed in order to test the central $10^{\circ}$ surrounding fixation, slightly above the threshold [18, $32]$. For example, Wall and colleagues $[18,32]$ proposed the use of cross-polarizing filters placed in front of the patient's eye in order to vary the luminance (a low-lumi- nance condition) of the white Amsler grid on a black background. By rotating the cross-polarizing filters, patients can view the target with the grid barely perceptible. Normal subjects may fail to see the corners of the grid, but do not report other defects [32]. Therefore, Wall and Sadun [32] recommended the use of threshold Amsler grid for metamorphopsia detection in the central $10^{\circ}$ of visual field.

\section{Modified Amsler Charts}

Some modified grid tests have been proposed even if limited in use. The Modified Amsler Chart was proposed by Shinoda et al. [46] in 2000 to test the macular area and detect lesions larger than 1 disc diameter by evaluating metamorphopsia in patients with epiretinal membrane. It consists of a grid of black lines on a white background with external dimensions of $12 \times 12 \mathrm{~cm}\left(24^{\circ}\right.$ high and $24^{\circ}$ wide with $12^{\circ}$ on each side of the center) and was comprised of individual grid squares measuring $2 \times 2 \mathrm{~cm}$. Each small square subtends an angle of $4^{\circ}$ and projects onto approximately $1.1 \mathrm{~mm}^{2}$ of the fundus [46]. In patients with metamorphopsia, the length of the lines is increased both in the vertical as in the horizontal direction compared to straight line, thus indicating the severity [46]. This test requires significant patient understanding and collaboration, as they are asked to draw manually the lines.

Bouwens and Van Meurs [47] proposed a set of eight modified Amsler charts called Sine Amsler Charts (SACs) to evaluate metamorphopsia in patients with macular pucker. In SACs, the straight lines in the center of the standard Amsler chart were replaced by sinusoid lines with a constant frequency but increasing amplitudes, resulting in representation of different grades of metamorphopsia, assigned from 1 to 8 [47]. On the different SACs, the lines have the form of $\mathrm{f}(\mathrm{x})=\mathrm{b}$.sinx, with increasing steps of the amplitude b of 0.25 of the previous amplitude in each following chart. The authors reported that SACs are a highly reproducible method (as patients easily reestablish the stepwise sequence of the charts and could easily recognize the previous choice) and easily understandable by the patients, requiring only a few minutes for determination of metamorphopsia [47]. The major limits of SACs include, as in the standard Amsler grid, the necessity of having reasonable reading vision in order to discern the lines, and moreover the presence of ERM in both eyes and the amblyopia in the unaffected eye [47]. 


\section{Differential Perimetry}

Differential perimetry was proposed by Jensen and Larsen [48] in 1998 to assess the amplitude of PR displacement. This technique is based on a theory that a local displacement of a group of PRs causes a shift in the visual field projection of the involved PRs. A healthy fellow eye and normal binocular vision are required, as in this way unilateral displacement of the PR will lead to loss of visual field correspondence with the cortically paired receptor element in the fellow eye [48]. As the brain continues to assign the original visual field projection to a stimulus reaching the displaced receptor, metamorphopsia, micropsia, and macropsia are all induced by the discrepancy between real and perceived visual field projection. Differential perimetry was performed with Goldmann kinetic perimetry (50 $\mathrm{cm}$ working distance), using red and green filter glasses, black binocular fixation targets, and red and green selective monocular stimuli (Goldmann III and IV) with fundus image superimposition of perimetry data [48]. The authors reported that macular holes are accompanied by metamorphopsia, micropsia, and central scotoma due to the presence of radial centrifugal PR displacement away from the center of the retina [48]. As stated by the authors, differential perimetry was shown to be a valid and objective test for accurate and detailed study of macular diseases characterized by mechanical attenuation or crowding of PRs, such as macular holes or puckers [48].

\section{M-CHARTS}

Matsumoto et al. [49] proposed the M-CHARTS for quantification of severity of metamorphopsia. This method is based on the fact that if a straight line is replaced with a dotted line and the dot interval is changed from fine to coarse, the distortion of the line decreases with increasing dot interval until eventually the dotted line appears straight $[49,50]$. M-CHARTS consist of 19 dotted lines with dot intervals ranging from $0.2^{\circ}$ to $2^{\circ}$ visual angles [50]. There are two types of M-CHARTS: type 1 with one dotted line and type 2 with two dotted lines on each chart, used for patients with central scotoma. The examination distance is set at $30 \mathrm{~cm}$ and fixation target is $0.3^{\circ}$. Firstly, a vertical straight line is presented to the patient. If it is recognized as straight, the score is 0 . If the patient recognizes the straight line as irregular or curved, then the following charts with dotted lines where the interval changes from fine to course are shown one after another.
When the patient recognizes the dotted line as straight, its visual angle is considered and their metamorphopsia scores. Thereafter, M-CHARTS are rotated $90^{\circ}$ and the same test is performed using horizontal lines. The authors reported that the simplicity of just answering if the line is distorted or not is one of the advantages of $\mathrm{M}$ CHARTS [49]. Metamorphopsia contains different frequency components of distortion, such as high-frequency (usually observed in early stages of ERM and detected by fine dotted lines) and low-frequency components (observed in advanced stages of ERM, detected by all kinds of lines including coarse dotted lines) [49].

\section{Preferential Hyperacuity Perimeter}

In order to address some of the shortcomings of the previous examinations (e.g. unrealiability due to unstable fixation, cortical image completion or filling in of scotoma, and 'visual crowding' effect) a new method, using hyperacuity function, was developed to evaluate the central visual field. Hyperacuity, also called 'Vernier acuity', is defined as the ability to perceive a minute difference in the relative spatial localization of two or more visual stimuli $[51,52]$. The hyperacuity threshold may be as low as 3-6 s of arc. The hypothesis on which preferential hyperacuity perimeter (PHP) examination is based is that when a dotted line is presented to a healthy retina, the corresponding collinear line of PR field is stimulated, the information is processed by the visual cortex, and a straight dotted line is perceived [53]. In case of retinal elevation (drusen, pigment epithelial detachment, etc.), there is a geometric shift in the PR location with consequent stimulation of a misaligned PR field, and a straight dotted line may be perceived at a different location from the true location in space [53]. This perceived shift in object location may explain metamorphopsia and is recorded by PHP, as proposed by Loewenstein et al. [54]. In fact, these authors reported greater sensitivity and positive predictive value of PHP versus the Amsler grid in evaluating AMD-related lesions [54].

\section{Shape Discrimination Hyperacuity Test}

Wang et al. [55] developed a shape discrimination hyperacuity (SDH) test with perfect and distorted circular contours called radial frequency (RF) patterns. It is based on the fact that humans have very high sensitivity to sinusoidal deformation from circularity [56]. The thresh-
30

Ophthalmic Res 2016;55:26-36 DOI: $10.1159 / 000441033$
Midena/Vujosevic 
old for detecting radial deformation is a hyperacuity $(<10$ arcsec) and is not affected by contrast reduction (for contrast $>10 \%$ ) at low RFs and shows little change with normal aging [56-58]. SDH has been shown to be significantly reduced in patients with AMD, even though patients still had normal vision [55]. The radial deformation is introduced by sinusoidally modulating the radius. The main parameters describing an RF pattern include mean radius (the radius of the undeformed RF pattern), the number of modulation cycles per circumference (RF), amplitude of radial modulation (the amount of deformation), peak spatial frequency of RF patterns (determining the width of the contour), and stimulus contrast [55]. It has been suggested that global visual integration is required to achieve optimal performance $[56,59]$. In this way, by measuring shape discrimination hyperacuity, a patient's ability to detect visual distortion and to integrate visual information can be quantified. The test is performed on a computer with specific standard characteristics.

\section{Home Monitoring Tests}

With an increasing need for reducing the ophthalmologists' burden in the management of patients with maculopathies, remote monitoring of disease and the use of telemedicine have become an increasing worldwide need. Thus, several home-monitoring tests and mobile handheld devices have been recently proposed.

\section{Home-Monitoring Devices Based on PHP}

The ForeseeHome home-testing device (Notal Vision Ltd., Tel Aviv, Israel) for home testing was approved by the Food and Drug Administration in 2009 [60], and was developed to detect visual acuity disturbances associated with choroidal neovascularization (CNV) due to AMD prior to overt visual symptoms [60]. The ForeseeHome monitoring device tests the central area of visual field of $14^{\circ}$ with approximately 500 data points sampled rapidly 3-5 times [60]. ForeseeHome is a stand-alone device designed for placement on a desk or tabletop. It is connected to a power outlet and phone line, and does not require a computer or Internet connection. Briefly, this technique stimulates a dot deviation signal flashed in a pseudorandom predetermined order. During the course of the exam, artificial distortion is presented to the patient (similar to that seen by patients with neovascular AMD). When a signal is presented to the retina over a CNV lesion, the pathological distortion and the artificial distor- tion compete for the patient's attention, and preferential looking will direct the patient to select the more extreme distortion. The patient should indicate the distortion along the dotted line with a computer mouse. Varying amplitudes of the artificial distortions serve as control stimuli to quantify the extent of eventual pathologic distortions correlated to retinal pathology. The intensity of the visual field defect is determined based on a grayscale map. Data obtained from patients are compared to a normative database, and the system determines whether there are visual responses which may be associated with progression of AMD to CNV [60]. The sensitivity and specificity of the ForeseeHome device in distinguishing between newly diagnosed $\mathrm{CNV}$ and intermediate AMD in an unsupervised manner (at home) was on average $85 \%$, thus comparable to PHP used in a clinical setting [61]. Moreover, the sensitivity of the ForeseeHome device is not influenced by type, size, or location of the CNV lesion [61].

\section{Mobile Handheld Devices}

Mobile handheld devices have been implemented for use by patients at home for remote monitoring of SDH using a handheld SDH test (myVisionTrack; Vital Art and Science Inc., Richardson, Tex., USA) implemented on a mobile platform (iOS; Apple Inc., Cupertino, Calif., USA) [62]. The test is performed on an iPod (Apple) [63]. The patient should keep the device at a distance of about an arm's length and indicate by touch input which of 3 circular shapes on the iPod was distorted [63].

\section{Health Management Tool}

The Health Management Tool (HMT) is a portable, remote monitoring system using a mobile handheld device on which the HMT application is installed. Data input by patients are transferred in real time to the HMT database, which receives and monitors data and controls communications with patients and clinicians [64]. Clinicians can log in to the HMT database from an Internetbased dashboard to view results and compliance information [64]. HMT has a specific myVisionTrack (Vital Art and Science), an iOS-based (Apple) application installed to assess shape discrimination hyperacuity [62]. Kaiser et al. [64] validated in a small population the feasibility of the HMT system in elderly patients with neovascular AMD. They found that with appropriate training and guidance in the use of mobile handheld devices, there was a very high compliance $(98.9 \%)$ with at least weekly monitoring and daily compliance exceeding $80 \%$ in visual function testing. 


\section{Metamorphopsia in Age-Related Macular Degeneration}

Nowomiejska et al. [65] compared the results of MCHARTS and the Amsler grid with OCT in the assessment of metamorphopsia in patients with neovascular AMD treated with bevacizumab. This study reported a higher rate of metamorphopsia detection with $\mathrm{M}$ CHARTS (89\%) versus the Amsler grid (69\%) [65]. Moreover, a significant change was found only in the median decrease of the horizontal metamorphopsia score from $0.3^{\circ}$ to $0.2^{\circ}$ after bevacizumab treatment, and no correlation was found between the degree of metamorphopsia and visual acuity or central retinal thickness [65].

In a recent systematic review and meta-analysis of studies examining the diagnostic value of the Amsler grid and PHP tests to rule out neovascular AMD in the screening setting, Faes et al. [66] reported the pooled sensitivity of 0.78 (95\% CI: $0.64-0.87$ ) and pooled specificity of 0.97 (95\% CI: 0.91-0.99) of the studies assessing the Amsler grid. The corresponding positive and negative likelihood ratios were 23.1 (95\% CI: 8.4-64.0) and 0.23 (95\% CI: $0.14-0.39)$, respectively. The pooled sensitivity of studies assessing the PHP was 0.85 (95\% CI: $0.80-0.89$ ), and specificity was 0.87 (95\% CI: 0.82-0.91). The corresponding positive and negative likelihood ratios were 6.7 (95\% CI: 4.6-9.8) and 0.17 (95\% CI: 0.13-0.23) [66]. Recently, the Home Monitoring of the Eye (HOME) Study, a phase 3 unmasked randomized trial, evaluated the role of home monitoring with the ForeseeHome device with telemonitoring plus standard care compared to standard care alone for eyes at risk of developing CNV due to AMD, and thus the possibility of an earlier diagnosis of $\mathrm{CNV}[60$, 67]. Patients randomized to the device arm had significantly better visual acuity at the time of detection of incident $\mathrm{CNV}$ when compared with those randomized to standard care only [67]. The authors concluded that persons at high risk for CNV development benefit from the home-monitoring strategy, which may increase the likelihood of better visual acuity results after intravitreal antiVEGF therapy [67].

The studies that evaluated shape discrimination hyperacuity, specifically on handheld mobile devices, showed that these tests, which are easy to use in patients with AMD with comparable results to those performed on personal computers, are sensitive to the severity of maculopathy $[63,64]$. In fact, in patients with advanced AMD, handheld shape discrimination hyperacuity was significantly reduced when compared with that of patients with high-risk early AMD [63].

\section{Metamorphopsia in Vitreoretinal Interface Disorders}

Metamorphopsia is a common symptom in ERM, macular holes, retinal detachment, and even after successful retinal surgery [13, 46-49, 68-75]. Shinoda et al. [46] correlated the severity of metamorphopsia determined with modified Amsler charts with the subjective score in patients with ERM. Metamorphopsia was more severe in the horizontal than in the vertical direction [46]. Bouwens and Van Meurs [47] reported that SACs were a reproducible method for evaluating metamorphopsia in patients undergoing surgery for macular pucker, with a decrease in metamorphopsia alone in $82 \%$ of patients, and a decrease in metamorphopsia associated with an increase in visual acuity in $43 \%$ of patients. Matsumoto et al. [49] reported that in patients with ERM, the increase in metamorphopsia score (determined with M-CHARTS) depends on the severity of membrane proliferation classified by scanning laser opthalmoscope images. The authors discussed the importance of the frequency component of distortion in metamorphopsia when evaluating ERM [49]. In the early stages of ERM, a high-frequency component of metamorphopsia is usually observed and detected by fine dotted lines and not by coarse dotted lines. Instead, in advanced stages of ERM, the large amplitude and low-frequency components of metamorphopsia are increased; therefore, all kinds of lines can detect metamorphopsia. An increasing contraction of the neurosensory retina was correlated with increasing metamorphopsia score (MCHARTS) over 3 years in patients with ERM [68]. Specifically, more severe vertical retinal contraction correlated with increasing severity of horizontal metamorphopsia, and more severe horizontal retinal contraction correlated with increasing severity of vertical metamorphopsia [68] (fig. 1). Moreover, in patients undergoing surgery for ERM, there was a different time course in improvement of vertical and horizontal metamorphopsia, as determined by M-CHARTS [69]. Whereas the mean horizontal metamorphopsia score continued to improve up to 12 months after surgery, the mean vertical score reached a plateau at 6 months after surgery [69]. The severity in preoperative metamorphopsia and central foveal thickness were significantly correlated to the degree of postoperative metamorphopsia. And disruption of the inner segment/outer segment junction of more than 200 microns was found to be a negative prognostic factor for metamorphopsia outcome (determined with PHP) after surgery for idiopathic ERM [70]. Moreover, Watanabe et al. [13] showed that metamorphopsia (determined with the Amsler chart) was detected in the same position as the
32

Ophthalmic Res 2016;55:26-36 DOI: $10.1159 / 000441033$
Midena/Vujosevic 

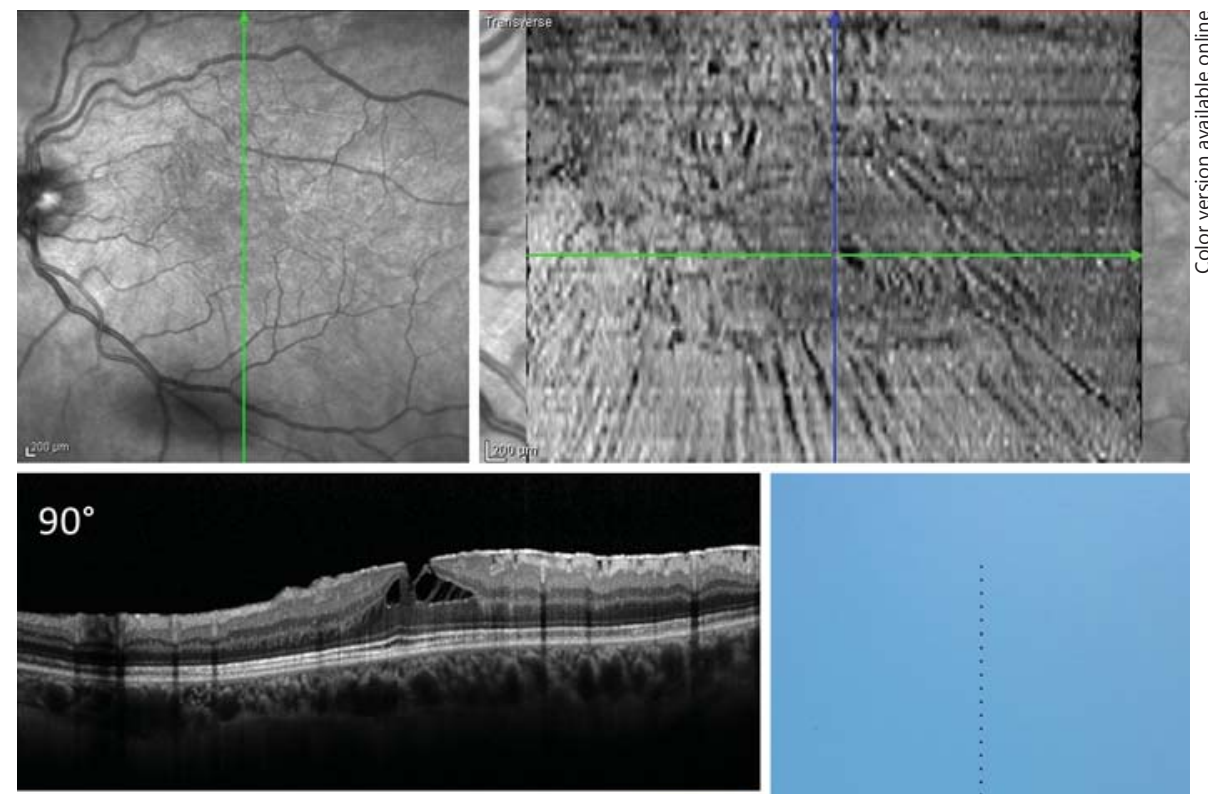

Fig. 1. Left eye of a patient with vitreoretinal interface syndrome characterized by epiretinal membrane and lamellar macular hole, who presented good visual acuity (20/20) but complained of important metamorphopsia that precluded her work activity as video terminalist and wanted to undergo surgical treatment. Infrared fundus image, linear spectral domain OCT scan, and en face OCT image showing distortion of the inner retinal surface with tangential epiretinal traction in the fovea and formation of a lamellar macular hole. The outer retina is preserved. The M-CHARTS show a horizontal $\mathrm{M}$ score of $0.8^{\circ}$ and a vertical $\mathrm{M}$ score of $0.5^{\circ}$, confirming the metamorphopsia perceived by the patient.
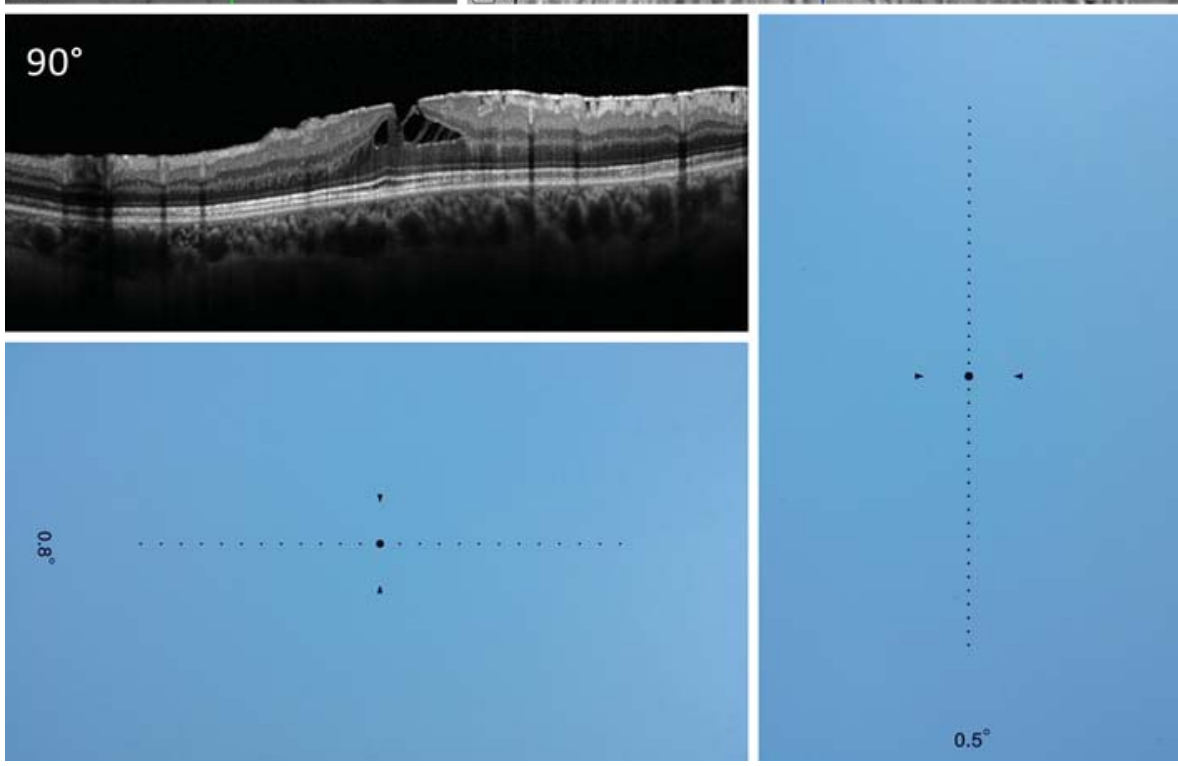

edematous area of the INL with thickness $>50 \mu \mathrm{m}$; therefore, mapping INL thickness with SD-OCT may be a useful and objective method for determining the position of metamorphopsia.

In idiopathic macular holes, metamorphopsia, micropsia, and central scotoma (extending outside the rim of the hole) have been reported $[48,71]$. Arimura et al. [72] found a significant correlation between metamorphopsia score determined with M-CHARTS and fluid cuff size (shallow retinal detachment), but not with the diameter of the macular hole. No significant relationship was found between metamorphopsia scores and visual acuity [72]. Usually after surgery for a macular hole, a decrease in metamorphopsia was reported [73, 74]. Krøyer et al. [73] suggested that both tangential displacement of the PRs and the eversion of the PRs (due to inner margin eversion) are responsible for metamorphopsia. These phenomena may explain the persistence of metamorphopsia after successful surgical closure of the hole (elimination of eversion and thus closure of the hole, yet per- sistence of residual tangential displacement) [73]. Moreover, the shape of the fovea after surgical closure of the macular hole, specifically the elongation asymmetry of the foveal tissue, was correlated to the degree of metamorphopsia as determined by M-CHARTS [75]. This was probably due to the fact that asymmetric elongation of foveal tissue prevented recovery of displaced PRs by inducing asymmetric tangential traction [75]. Although the asymmetric elongation was mostly found in the INL and outer nuclear layer rather than in the PR layer, the traction of specific retinal layers may induce some PR displacement and thus metamorphopsia [75]. Another explanation could be that asymmetric elongation and stretching of the retinal layers prevented a proper signal transduction [75]. In fact, differential perimetry showed radial centrifugal PR displacement in patients with an idiopathic macular hole, away from the center of the retina [48].

In patients with macula-off retinal detachment, metamorphopsia was more severe than in patients with mac- 
ula-on retinal detachment [76]. The incidence of metamorphopsia after surgery for retinal detachment was reported to be up to $67 \%$ [77]. The most frequent OCT findings in patients with metamorphopsia were presence of ERM, disruption of the inner segment/outer segment, cystoid macular edema, macular hole, or subretinal fluid, even though no OCT retinal changes could be detected after rhegmatogenous retinal detachment surgery in 32 out of 50 eyes with metamorphopsia [76]. Rossetti et al. [78] also reported that long-standing metamorphopsia could occur after successful macula-off rhegmatogenous retinal detachment repair even without detectable signs of PR disruption on OCT. The persisting metamorphopsia appeared to come from retinal vertical displacement by rhegmatogenous retinal detachment surgery [76].

\section{Metamorphopsia in Diabetic Retinopathy}

It is well established that visual function can be decreased in diabetic patients even without any clinical sign of diabetic retinopathy [79]. Decreased foveal function may be evaluated with contrast sensitivity, threshold Amsler grid, and more recently with microperimetry [79]. The presence of metamorphopsia was evaluated by Wall et al. [25] using 4 different types of Amsler grids (low-intensity grids) in diabetic patients without and with background diabetic retinopathy. They found the standard white grid was the best for detecting metamorphopsia, whereas the yield of scotoma was the highest with the threshold Amsler grid [25]. Moreover, low-intensity Amsler grids improved the sensitivity of Amsler grid testing in diabetics without diabetic retinopathy [25]. Wolfe and Sadun [27] documented that the threshold Amsler grid was approximately 5-fold more sensitive than the standard Amsler grid in evaluating metamorphopsia and relative scotoma in diabetic patients with macular edema. The threshold Amsler grid test proved to have $100 \%$ sensitivity in the eyes in which metamorphopsia was found by conventional Amsler grid, thus demonstrating that relative scotomas were present in the same area as in the threshold Amsler grid [25].

\section{Metamorphopsia in Central Serous Chorioretinopathy}

Blurring of vision, central scotoma, hypermetropization, and a variable level of metamorphopsia and micropsia are common complaints of patients with CSC [80].
Sometimes visual disturbances persist despite resolution of subretinal foveal detachment. Bae and Chae [80] evaluated metamorphopsia by M-CHARTS and correlated the degree of metamorphopsia with OCT findings in patients with CSC. Fifteen (45.5\%) out of 33 examined patients had metamorphopsia. No correlation was found between the extent of metamorphopsia and posterior clumping of the PR layer, high reflectivity of the PR layer, or large-scale neuroretinal detachment. Only focal retinal pigment epithelium detachment was more common in patients with metamorphopsia than in those without metamorphopsia. There were no correlations between metamorphopsia scores and visual acuity data, probably due to different durations of CSC, or to the limits of the M-CHARTS. Therefore, the authors concluded that MCHARTS could be useful as an adjunct to OCT for evaluation of the clinical course of CSC.

\section{Conclusions}

Unfortunately, with the advancements in retinal imaging technology, increasing discrepancy is evident between detailed structural evaluation of specific retinal/ choroidal lesions and visual function, represented by simple visual acuity quantification. Clinicians mostly monitor treatment results of macular disorders with morphologic examination (mainly OCT), whereas the patient's perspective is to gain (or stabilize) vision, both quantitatively and qualitatively. Moreover, it seems important to correlate morphologic aspects of the retina/choroid to the real patient's complaints. Therefore, a detailed visual function evaluation, including different aspects of vision (not just mere visual acuity) as well as different qualitative aspects (metamorphopsia), may help to better understand macular disorders, the results of standard treatments, and the relevance of earlier treatment. The importance of the patient's perspective is very well illustrated by the recent technical appraisal by NICE (UK) for ocriplasmin reimbursement for vitreomacular traction in UK [81]. According to this regulatory authority, National Health System reimbursement of a new drug is allowed if the quality-adjusted life year value is under a certain preplanned cutoff value. In evaluating ocriplasmin for vitreomacular traction, the importance of patients' opinion is clearly documented by the following passage: 'The Committee considered the impact of VMT on the everyday life of patients. It heard from the patient expert about the problems associated with VMT, including difficulties with reading, cooking, watching television and driving
Midena/Vujosevic 
(metamorphopsia is included) that prevented them from enjoying these activities. The Committee concluded that resolving VMT without the need for surgery would be beneficial to the wellbeing of patients with VMT.' And in patients with vitreomacular traction, metamorphopsia is one of the most disabling visual symptoms.

In conclusion, even with the current limitations in quantifying it, quantitative evaluation of metamorphopsia appears as an essential step in quantifying visual func- tion in patients with macular disorders, and an essential step in evaluating treatment results (from the patient's point of view) with the available techniques.

\section{Disclosure Statement}

The authors declare no conflicts of interest.

\section{References}

1 Wiecek E, Lashkari K, Dakin SC, Bex P: Novel quantitative assessment of metamorphopsia in maculopathy. Invest Ophthalmol Vis Sci 2014;56:494-504.

2 Amsler M: L'Examen qualitatif de la fonction maculaire. Ophthalmologica 1947;114:248261.

3 Forster R: Metamorphopsie. Ein Symptom partieller Schrumpfung der Retina (Retinitis circumscripta). Berlin, Ophthalmologische Beiträge, 1862.

4 Knapp H: Ueber isolirte Zerreissungen der Aderhaut in Folge von Traumen auf dem Augapfel. Arch Augenheilk 1869;1:6-29.

5 Wundt W: Zur Theorie der raumlichen Gesichtswahrnehmungen. Philosophische Studien 1898; $14: 1$.

6 Amsler M: Quantitative and qualitative vision. Trans Ophthalmol Soc UK 1949;69: 397-410.

7 Amsler M: Earliest symptoms of diseases of the macula. Br J Ophthalmol 1953;37:521537.

8 Mackay G: On blinding of the retina by direct sunlight; a study in prognosis. Ophthal Rev 1984;13:1-20, 41-57, 83-96.

9 Oguchi C: Eine besondere Form von Chorioretinitis centralis nebst Bemerkungen über nachbildartiges Skotom. Albrecht von Graefes Archive für Ophthalmologie 1922;110:25.

10 Kitahara S: Ueber klinische Beobachtungen bei der in Japan häufig vorkommenden Chorioretinitis centralis serosa. Klin Mbl Augenheilk 1936;97:345.

11 Horniker A: Su di una forma di retinite centrale di origine vasoneurotica. Ann Ottal 1927;55:578.

12 Bruckner R, Field NH: Beitrag zum Symptomenkomplex der Chorioretinitis centralis serosa. Ophthalmologica 1945;109:281.

13 Watanabe A, Arimoto S, Nishi O: Correlation between metamorphopsia and epiretinal membrane optical coherence tomography findings. Ophthalmology 2009;116:1788-1793.

14 Okamoto F, Sugiura Y, Okamoto Y, Hiraoka T, Oshika T: Associations between metamorphopsia and foveal microstructure in patients with epiretinal membrane. Invest Ophthalmol Vis Sci 2012;53:6770-6775.
15 Ponsioen TL, Hooymans JL, Hols LI: Remoddeling of the human vitreous and vitreoretinal interface-a dynamic process. Progr Retin Eye Res 2010;29:580-595.

16 Agte S, Junke S, Matthias S, Ulbricht E, Erdmann I, Wurm A, Schild D, Kas JA, Reichenbach A: Müller glial cell-provided cellular light guidance through the vital guinea pig retina. Biophys J 2011;101:2611-2619.

17 Schuchard RA: Validity and interpretation of Amsler grid reports. Arch Ophthalmol 1993; 111:776-780.

18 Wall M, May DR: Threshold Amsler grid testing in maculopathies. Ophthalmology 1987; 94:1126-1133.

19 Boldrey EE: Foveal ablation for subfoveal choroidal neovascularization. Ophthalmology 1989;96:1430-1436.

20 Walsh AW, Magargal LE, Wright F, Donoso LA: The early natural history of subfoveal neovascular membranes in eyes with age-related macular degeneration. Ann Ophthalmol 1989;21:348-350.

21 Swann PG, Lovie-Kitchin JE: Age-related maculopathy. II: the nature of the central visual field loss. Ophthalmic Physiol Opt 1991; 11:59-70.

22 Natsikos VE, Dean Hart JC: Static perimetric and Amsler chart changes in patients with idiopathic central serous retinopathy. Acta Ophthalmol 1980;58:908-917.

23 Easterbrook M: The use of Amsler grids in early chloroquine retinopathy. Ophthalmology 1984;91:1368-1372.

24 Easterbrook M: The sensitivity of Amsler grid testing in early chloroquine retinopathy. Trans Ophthalmol Soc UK 1985;104:204207.

25 Wall M, Collins C, May DR: Low-intensity grids improve sensitivity of Amsler grid testing in diabetic patients without background retinopathy. Ann Ophthalmol 1990;22:96-100.

26 Ruiz RS, Saatci OA: Chloroquine and hydroxychloroquine retinopathy: how to follow affected patients. Ann Ophthalmol 1991;23: 290-291.

27 Wolfe KA, Sadun AA: Threshold Amsler grid testing in diabetic retinopathy. Graefes Arch Clin Exp Ophthalmol 1991;229:219-223.
28 Bernstein HN: Ocular safety of hydroxychloroquine. Ann Ophthalmol 1991;23:292-296.

29 Zygulska-Mach H, Starzycka M, Ciechanowska A: Retinal function after surgical treatment of detachment. Ophthalmologica 1979; 178:210-214

30 Kreissig I, Lincoff H, Witassek B, Kolling G: Color vision and other parameters of macular function after retinal reattachment. Dev Ophthalmol 1981;2:77-85.

31 Smith RG, Hardman Lea SJ, Galloway NR: Visual performance in idiopathic macular holes. Eye 1990;4:190-194.

32 Wall M, Sadun AA: Threshold Amsler grid testing. Cross-polarizing lenses enhance yield. Arch Ophthalmol 1986;104:520-523.

33 Yannuzzi LA: A modified Amsler grid. A selfassessment test for patients with macular disease. Ophthalmology 1982;89:157-159.

34 Grand MG, Burgess DB, Singerman LJ, Ramsey J: Choroidal osteoma. Retina 1984;4:84-89.

35 Fine SL: Early detection of extrafoveal neovascular membranes by daily central field evaluation. Ophthalmology 1985;92:603-609.

36 Folk JC: Aging macular degeneration. Ophthalmology 1985;92:603-609.

37 Singerman LJ: Important points in management in patients with choroidal neovascularization. Ophthalmology 1985;92:610-614.

38 Fine AM, Elman MJ, Ebert JE, Prestia PA, Starr JS, Fine SL: Earliest symptoms caused by neovascular membranes in the macula. Arch Ophthalmol 1986;104:513-514.

39 Chang MA, Morgan CM, Schatz H: The Amsler grid. Retina 1987;7:279.

40 Morrison DL, Magargal LE, Ehrlich DR Goldberg RE, Robb-Doyle E: Review of choroidal osteoma: successful krypton red laser photocoagulation of an associated subretinal neovascular membrane involving the fovea. Ophthalmic Surg 1987;18:299-303.

41 Achard OA, Safran AB, Duret FC, Ragama E: Role of the completion phenomenon in the evaluation of Amsler grid results. Am J Ophthalmol 1995;120:322-329.

42 Parkes L, Lund J, Angelucci A, Solomon JA, Morgan M: Compulsory averaging of crowded orientation signals in human vision. Nat Neurosci 2001;4:739-744. 
43 Ramachandran VS, Gregory RL: Perceptual filling in of artificially induced scotomas in human vision. Nature 1991;350:699-702.

44 Heinen SJ, Skavenski AA: Recovery of visual responses in foveal VI neurons following bilateral foveal lesions in adult monkey. Exp Brain Res 1991;83:670-674.

45 Gilbert CD, Wiesel TN: Receptive field dynamics in adult primary visual cortex. Nature 1992;356:150-152.

46 Shinoda K, Ishida S, Kawashima S, Matsuzaki T, Yamada K, Katsura H: A new method for quantification of metamorphopsia in patients with epiretinal membrane. Jpn J Ophthalmol 2000;44:424-427.

47 Bouwens MD, Van Meurs JC: Sine Amsler Charts: a new method for the follow-up of metamorphopsia in patients undergoing macular pucker surgery. Graefes Arch Clin Exp Ophthalmol 2003;241:89-93.

48 Jensen OM, Larsen M: Objective assessment of photoreceptor displacement and metamorphopsia: a study of macular holes. Arch Ophthalmol 1998;116:1303-1306.

49 Matsumoto C, Arimura E, Okuyama S, Takada S, Hashimoto S, Shimomura Y: Quantification of metamorphopsia in patients with epiretinal membranes. Invest Ophthalmol Vis Sci 2003;44:4012-4016.

50 Arimura E, Matsumoto C, Nomoto H, Hashimoto S, Takada S, Okuyama S, Shimomura Y: Correlations between M-CHARTS and PHP findings and subjective perception of metamorphopsia in patients with macular diseases. Invest Ophthalmol Vis Sci 2011;52:128-135.

51 Westheimer G: The spatial sense of the eye. Invest Ophthalmol Vis Sci 1979;18:893-912.

52 Enoch JM, Williams RA, Essock EA, Barricks M: Hyperacuity perimetry. Assessment of macular function through ocular opacities. Arch Ophthalmol 1984;102:1164-1168.

53 Goldstein M, Loewenstein A, Barak A, Pollack A, Bukelman A, Katz H, Springer A, Schachat AP, Bressler NM, Bressler SB, Cooney MJ, Alster Y, Rafaeli O, Malach R Preferential Hyperacuity Perimeter Research Group: Results of a multicenter clinical trial to evaluate the preferential hyperacuity perimeter for detection of age-related macular degeneration. Retina 2005;25:296-303.

54 Loewenstein A, Malach R, Goldstein M, Leibovitch I, Barak A, Baruch E, Alster Y, Rafaeli O, Avni I, Yassur Y: Replacing the Amsler grid: a new method for monitoring patients with age-related macular degeneration. Ophthalmology 2003;110:966-970

55 Wang YZ, Wilson E, Locke KG, Edwards AO Shape discrimination in age-related macular degeneration. Invest Ophthalmol Vis Sci 2002;43:2055-2062.

56 Wilkinson F, Wilson HR, Habak C: Detection and recognition of radial frequency patterns. Vision Res 1998;38:3555-3568.

57 Hess RF, Wang YZ, Demanins R, Wilkinson F, Wilson HR: A deficit in amblyopia for global shape detection. Vision Res 1999;39:901914.
58 Wang YZ: Effects of aging on shape discrimination. Optom Vis Sci 2001;78:447-454.

59 Hess RF, Wang YZ, Dakin SJ: Are judgments of circularity local or global? Vision Res 1999; 39:4354-4360.

60 Chew EY, Clemons TE, Bressler SB, Elman MJ, Danis RP, Domalpally A, Heier JS, Kim JE, Garfinkel RA; Appendix 1 for AREDS2HOME Study Research Group: Randomized trial of the ForeseeHome monitoring device for early detection of neovascular age-related macular degeneration. The Home Monitoring of the Eye (HOME) study design - HOME Study report number 1 . Contemp Clin Trials 2014;37:294-300.

61 Loewenstein A, Ferencz JR, Lang Y, Yeshurun I, Pollack A, Siegal R, Lifshitz T, Karp J, Roth D, Bronner G, Brown J, Mansour S, Friedman S, Michels M, Johnston R, Rapp M, Havilio M, Rafaeli O, Manor Y: Toward earlier detection of choroidal neovascularization secondary to age-related macular degeneration: multicenter evaluation of a preferential hyperacuity perimeter designed as a home device. Retina 2010;30:1058-1064.

62 Chhetri AP, Wen FY, Wang YZ, Zhang K: Shape discrimination test on handheld devices for patient self-test; in Veinot T (ed): Proceedings of the First Annual ACM International Health Informatics Symposium (IHI 2010). Arlington, ACM Press 2010, pp 502 506.

63 Wang YZ, He YG, Mitzel G, Zhang S, Bartlett M: Handheld shape discrimination hyperacuity test on a mobile device for remote monitoring of visual function in maculopathy. Invest Ophthalmol Vis Sci 2013;54:5497-5505.

64 Kaiser PK, Wang YZ, He YG, Weisberger A Wolf S, Smith CH: Feasibility of a novel remote daily monitoring system for age-related macular degeneration using mobile handheld devices: results of a pilot study. Retina 2013; 33:1863-1870.

65 Nowomiejska K, Oleszczuk A, Brzozowska A, Grzybowski A, Ksiazek K, Maciejewski R, Ksiazek P, Juenemann A, Rejdak R: M-charts as a tool for quantifying metamorphopsia in age-related macular degeneration treated with the bevacizumab injections. BMC Ophthalmol 2013;13:13.

66 Faes L, Bodmer NS, Bachmann LM, Thiel MA, Schmid MK: Diagnostic accuracy of the Amsler grid and the preferential hyperacuity perimetry in the screening of patients with age-related macular degeneration: systematic review and meta-analysis. Eye (Lond) 2014; 28:788-796.

67 AREDS2-HOME Study Research Group, Chew EY, Clemons TE, Bressler SB, Elman MJ, Danis RP, Domalpally A, Heier JS, Kim JE, Garfinkel R: Randomized trial of a home monitoring system for early detection of choroidal neovascularization home monitoring of the Eye (HOME) study. Ophthalmology 2014;121:535-544

68 Arimura E, Matsumoto C, Okuyama S, Takada S, Hashimoto S, Shimomura Y: Retinal contraction and metamorphopsia scores in eyes with idiopathic epiretinal membrane. Invest Ophthalmol Vis Sci 2005;46:2961-2966.

69 Kinoshita T, Imaizumi H, Okushiba U, Miyamoto H, Ogino T, Mitamura Y: Time course of changes in metamorphopsia, visual acuity, and OCT parameters after successful epiretinal membrane surgery. Invest Ophthalmol Vis Sci 2012;53:3592-3597.

70 Bae SH, Kim D, Park TK, Han JR, Kim H, Nam W: Preferential hyperacuity perimeter and prognostic factors for metamorphopsia after idiopathic epiretinal membrane surgery. Am J Ophthalmol 2013;155:109-117.

71 Varano M, Scassa C, Capaldo N, Sciamanna M, Parisi V: Development of macular pseudoholes: a 36-month period of follow-up. Retina 2002;22:435-442.

72 Arimura E, Matsumoto C, Okuyama S, Takada S, Hashimoto S, Shimomura Y: Quantification of metamorphopsia in a macular hole patient using M-CHARTS. Acta Ophthalmol Scand 2007;85:55-59.

73 Krøyer K, Christensen U, la Cour M, Larsen M: Metamorphopsia assessment before and after vitrectomy for macular hole. Invest Ophthalmol Vis Sci 2009;50:5511-5515.

74 Fukuda S, Okamoto F, Yuasa M, Kunikata T, Okamoto Y, Hiraoka T, Oshika T: Vision-related quality of life and visual function in patients undergoing vitrectomy, gas tamponade and cataract surgery for macular hole. Br J Ophthalmol 2009;93:1595-1599.

75 Kim JH, Kang SW, Park DY, Kim SJ, Ha HS Asymmetric elongation of foveal tissue after macular hole surgery and its impact on metamorphopsia. Ophthalmology 2012;119:21332140 .

76 Okamoto F, Sugiura Y, Okamoto Y, Hiraoka T, Oshika T: Metamorphopsia and optical coherence tomography findings after rhegmatogenous retinal detachment surgery. Am J Ophthalmol 2014;157:214-220.

77 Wang Y, Li SY, Zhu M, Chen SJ, Liu Y, Men XH, Gillies M, Larsson J: Metamorphopsia after successful retinal detachment surgery: an optical coherence tomography study. Acta Ophthalmol Scand 2005;83:168-171.

78 Rossetti A, Doro D, Manfrè A, Midena E: Long-term follow-up with optical coherence tomography and microperimetry in eyes with metamorphopsia after macula-off retinal detachment repair. Eye (Lond) 2010;24:18081813.

79 Midena E, Vujosevic S: Visual psychophysics in diabetic retinopathy; in Tombran-Tink J, Barnstable CJ, Gardner TW (eds): Visual Dysfunction in Diabetes. Ophthalmology Research. New York, Springer, 2012, pp 69-103.

80 Bae SW, Chae JB: Assessment of metamorphopsia in patients with central serous chorioretinopathy. Indian J Ophthalmol 2013;61: 172-175.

81 NICE Technology Appraisal Guidance 297: Ocriplasmin for Treating Vitreomacular traction. London, NICE, 2013. www.guidance. nice.org.uk/ta297. 